\title{
Capítulo 6 \\ YouTube como objeto de investigación en la comunicación
}

\author{
Angel Torres-Toukoumidis \\ Universidad Politécnica Salesiana, Ecuador \\ Orcid: https://orcid.org/oooo-0oo2-7727-3985 \\ Isidro Marín-Gutiérrez \\ Universidad Técnica Particular de Loja, Ecuador \\ Orcid: https://orcid.org/oooo-0oo2-6858-0983 \\ DOI: https://doi.org/ 10.16921/ciespal.14.8
}

\section{Resumen}

El portal de Internet YouTube tiene más de 2000 millones de usuarios y preferentemente procede de dispositivos móviles como tabletas oteléfonos inteligentes. La necesidad de obtener opinión pública sobre temas diversos está muy extendida en las ciencias sociales y en comunicación en particular. Los métodos tradicionales basados en encuestas y en entrevistas o grupos de discusión están siendo reemplazados en parte por la extracción de datos de Internet. El potencial como herramienta de investigación de YouTube es poco conocido. Este capítulo de libro investiga este problema mediante la introducción y evaluación crítica de una estrategia sistemática de recopilación de información y análisis en YouTube. Ofrecemos el conocimiento de diversos programas gratuitos y webs que facilitan la extracción de datos de YouTube sin llegar a utilizar códigos de programación, minería de datos ni otras herramientas fuera de las competencias comunes del comunicador. Los resultados de estas investigaciones de comunicación en YouTube muestran que es posible identificar patrones de consumo audiovisual sobre diferentes temas. 
Palabras clave: investigación; análisis de contenido; muestra; metodología; YouTube.

\begin{abstract}
The YouTube Internet portal has more than 2000 million users and preferably comes from mobile devices such as tablets or smartphones. The need to obtain public opinion on diverse topics is widespread in the social sciences and in communication. Traditional methods based on surveys and interviews or discussion groups are being replaced in part by the extraction of data from the Internet. The potential as a YouTube research tool is little known. This book chapter investigates this problem by introducing and critically evaluating a systematic strategy of information gathering and analysis on YouTube. We offer the knowledge of various free programs and websites that facilitate the extraction of data from YouTube without using programming codes, data mining or other tools outside the common competencies of the communicator. The results of these communication research on YouTube show that it is possible to identify patterns of audiovisual consumption on different topics.
\end{abstract}

Keywords: research; content analysis, sample, methodology, YouTube.

Facebook, Twitter, Instagram y YouTube son plataformas principalmente enfocadas hacia el ocio y entretenimiento. La masiva producción de datos generados diariamente por las redes sociales ha despertado el interés de los académicos con el fin de establecer patrones, interacciones y soluciones que demarquen un desarrollo en la ciencia siendo esto posible observar en la educación, mercadotecnia, política, salud y la comunicación. Dentro del campo educativo las redes sociales han propiciado su incorporación como herramienta informal en el proceso de enseñanza-aprendizaje (Tan, 2013). De ahí que se ha motivado la suspicacia de los estudiantes respecto al material visualizado enfatizando la visión crítica hacia los nuevos medios (GarcíaRuiz, Pérez-Rodríguez \& Torres-Toukoumidis, 2018). En el campo del marketing las redes sociales han abierto una red posibilidades orientadas al patrocinio ( $\mathrm{Wu}, 2016)$ y experiencia del consumidor (Baron, Conway \& Warnaby, 2010). Se ha corroborado la influencia de 
las redes sociales en la democratización (Aguirre, Samaniego, Benítez, \& Marín-Gutiérrez, 2019) y especialmente en promover la participación e identidad de la ciudadanía (Loader \& Mercea, 2011). Respecto al tema de la salud se ha analizado su efecto en el monitoreo de enfermedades (Sarker et al., 2016) y en la difusión preventiva hacia epidemias y pandemias (Sharma, et al., 2017). Por último, las redes sociales también se han imbuido desde diferentes aristas en los estudios científicos de la comunicación, entre los que destacan los estudios del discurso crítico (Khosravinik \& Unger, 2016) y el análisis de la mediatización (Jansson y Lindell, 2018).

YouTube por sí sola también ha destacado en cada uno de los campos mencionados, verbigracia, en la educación se presenta como herramienta emergente para la educación anatómica (Jaffar, 2012; Burke, \& Snyder, 2008); para fomentar hábitos de lectura (RoviraCollado, 2017) o para dar una clase a aquellos alumnos que necesitan que les repitan los conceptos e ideas (Ruiz-San-Miguel, HinojosaBecerra, Marín-Gutiérrez, 2016). En la mercadotecnia etiquetan a YouTube desde la noción de herramienta, pero esta vez incorporado el branding (Chen, 2013) y especialmente la influencia de los YouTubers en el comportamiento del consumidor (Lopes et al., 2020). En cuanto a la política YouTube ha servido como espacio de transmisión de debates entre candidatos (Way, 2015) y formulación de campañas dirigidas al electorado joven (Bowyer, Kahne, \& Middaugh, 2017). En el campo de la salud, se prescribe mayoritariamente como fuente de información sobre el cuidado y protección (Madathil, et al., 2015). Igualmente, YouTube responde al contexto comunicativo en base a la capacitación de contenidos audiovisuales (Costa-Sánchez y Túñez-López, 2019) y divulgación de la cultura popular (Schneider, 2016).

Internet es una fuente de información y entretenimiento donde los usuarios buscan y comparten todo tipo de información al instante. En la Red podemos encontrar portales de información capaces de agilizar la transmisión de videos que se viralizan. Uno de las herramientas más utilizadas para difundir videos es YouTube que se define como la 
más popular comunidad de videos online del mundo y que permite a sus usuarios ver y compartir videos. YouTube se fundó en 2005. Son 1.900 millones de usuarios (representa casi un tercio de todo Internet). Los espectadores pasan más de 1000 millones de horas diarias en la plataforma y generan miles de millones de visualizaciones. Más del 70 \% del tiempo de visualización de YouTube procede de dispositivos móviles. Se encuentra en 91 países (en China o Corea del Norte está prohibido) y en 80 idiomas. Mil millones de horas reproducidas al día. El número de canales que ingresan más de 100.00o\$ anuales gracias a YouTube ha aumentado un $40 \%$ de un año a otro. El número de canales con más de un millón de suscriptores ha aumentado más de un 65\% de un año a otro (YouTube, 2020).

Esta capacidad de libre elección de los usuarios es muy útil para los estudiosos de la comunicación.

Existen múltiples estudios que describen el consumo televisivo tradicional aún hay poco sobre el consumo audiovisual a través de la Red. La televisión a nivel de países se limita a una muestra de paneles de audiometría en miles de diversos hogares (Kantar Media, 2013). Cuando analizamos una audiencia a través de Internet se realiza con la totalidad de los datos y, por tanto, es más certero (gracias a la cuantificación de las visitas, los tiempos de conexión a los videos, lugares desde donde se ve, etc...).

YouTube sí da beneficios gracias a la publicidad. Ofrece a las empresas grandes audiencias y clasificados en nichos (música, coches, películas, series, videojuegos, YouTubers, etc...). Gracias a YouTube podemos observar una amplia radiografía de gustos y tendencias a nivel nacional o internacional. YouTube intenta conocer a sus usuarios para ofrecerles una publicidad personalizada. En esta búsqueda por rentabilizar YouTube es necesario saber quiénes son sus usuarios y cuáles son sus gustos, opiniones e intereses. Esta información es importante para cualquier comunicólogo que se precie. El uso de YouTube está muy generalizado actualmente (Torres-Toukoumidis, Ramírez-Montoya, \& Romero-Rodríguez, 2018). 
Hay investigaciones que aseguran que Internet pone en peligro la identidad local. Lo global eliminará todo rastro de lo local. Fernández (2002) asegura que las grandes corporaciones globalizadoras eliminarán las culturas locales. O'Reilly (2006) afirma que Internet es un cerebro colectivo y que es el medio donde convergen el resto de medios. Pero ¿esto significa que las personas ya no consumen informaciones locales? Lo cierto es que hay una influencia de lo global pero tampoco se pierde lo singular de los aspectos locales y esto mismo podemos analizarlo con YouTube. Gracias a las herramientas que vamos a conocer podemos saber qué tipos de contenidos consumen los usuarios de YouTube. Burgess y Green (2009) afirman que YouTube es el nuevo campo de batalla por el poder del siglo XXI. Está siendo controlado por las industrias de medios de comunicación, por sus usuarios, por grupos de presión y por productores audiovisuales independientes.

¿Cómo son los usuarios de YouTube? Pérez y Santos (2009) aseguraban que los espectadores de YouTube interactuaban siempre. Dejan su rol de consumidores pasivos y comienzan a convertirse en generadores de contenidos (los llamados prosumidores). Díaz Arias (2009) nos indica que el video está comenzando a desarrollar lenguajes propios en Internet. Otros autores (Ritzer, 2001; Robison y Geofrey, 1997; Wolton, 2000; Owen, 2000) afirman que los usuarios de Internet tienen un rol pasivo como meros espectadores de contenidos audiovisuales en la Red. Quizás fuera cierto en la Web 1.o sobre la que escribieron pero no ocurre lo mismo en la Web 2.o ni en la Web 3.o. Así con las herramientas que vamos a ver podremos analizar las acciones sociales de YouTube (utilizar la función "Me gusta", "no me gusta", "compartir vídeos", "guardar", "favoritos" o "realizar comentarios"). Pero la realidad es que existe una baja interactividad por parte de los usuarios de YouTube. Bill Tancer (2008) estudió que menos del 1\% de las personas que visitan YouTube suben algún video.

En definitiva, YouTube contiene 1.900 millones de personas registradas. Esto implica que a nivel de contenido, forma y discurso se valora una heterogeneidad proclive a disponerse como una oportunidad 
para la investigación compartiendo una estructura sistematizada para la realización de artículos, libros, capítulos de libro y conferencias. Por ende, pudiendo ser utilizados por los estudiantes para sus trabajos de grado, fin de máster y doctorado. De hecho, este capítulo pretende demostrar una serie de estrategias pragmáticas legitimadas por la comunidad académica para realizar trabajos de investigación en el campo de la comunicación basados en diseños, muestras y softwares para el análisis de información extraída de la plataforma de videos.

\section{YouTube en la investigación de la comunicación}

\subsection{Diseño de investigación en comunicación que utilizan YouTube}

Tanto cuestionarios, entrevistas y grupos de discusión han sido métodos estándares de análisis en la ciencias sociales como en investigación en Comunicación. De ellos se han obtenido resultados que se han extrapolado al público, usuarios de servicios u otros grupos específicos. Así se han medido audiencias de programas radiofónicos o de televisión. Pero para la industria de la comunicación ahora su interés está en las redes sociales (Twitter o Facebook) y en concreto YouTube. El interés de los medios es buscar opiniones del público.

Actualmente se utilizan software de análisis de redes sociales que incluyen una combinación variada de herramientas de extracción de datos (Fan \& Gordon, 2014). En el mundo universitario, y en concreto en las facultades de Comunicación, hay un interés por crear métodos efectivos de análisis de las redes sociales.

Muchas técnicas de investigación social que existen se han adaptado para realizar análisis en Internet como el análisis de contenidos, el análisis etnográfico, el análisis de redes y sobre todo las encuestas (Tijdens \& Steinmetz, 2016). Los métodos cuantitativos se centran en contar ciertas actividades on line como son los tweets, los hashtag o los retweets en Twitter. Para YouTube se cuentan las palabras claves o los comentarios de los videos y se utilizan para analizar el 
interés concreto por un tema, también para analizar series temporales de tendencias. Estos enfoques cuantitativos utilizan algoritmos para generar nuevas ideas. Se trata de detectar conexiones entre usuarios y recursos y categorizarlos utilizando la polaridad de sentimientos (en el caso de YouTube contar los "me gusta" o "no me gusta") (Thelwall, Buckley y Paltoglou, 2011).

Estos softwares de análisis de redes sociales se han creado para permitir a los investigadores explorar los datos de diversas formas. Es necesario métodos combinados para ayudar a los investigadores en comunicación a seleccionar y evaluar estrategias de análisis apropiadas. YouTube es una fuente gratuita de información para los comunicólogos. Es el segundo sitio web más popular del mundo y está siendo utilizado por todas las compañías de comunicación del mundo (Fan y Gordon, 2014). Hasta el momento Twitter se analiza más en publicaciones académicas pero está limitado por la presencia de spam y de bots. YouTube es una fuente de información potencialmente valiosa sobre el hecho de ver videos, sobre sus usos y gratificaciones. Tiene un valor comunicacional fuera de toda duda (Lewis, Heath, Sornberger y Arbuthnott, 2012).

Se han realizados estudios académicos con YouTube con el análisis de contenido (Desai et al., 2013) pero lo normal es realizar combinación de métodos. Lo común es analizar las visualizaciones de series temporales para rastrear cambios. Un estudio realizado sobre campañas antitabaco analizó el éxito de seis videos antitabaco a través de los comentarios en YouTube y utilizó varias métricas (número de vistas, número de "me gusta" o "no me gusta"). También analizó las interacciones entre los comentaristas. Esta evaluación estaba basada en la evidencia del éxito de la campaña en Estados Unidos (Chung, 2015).

Los métodos de análisis de YouTube tienen los siguientes problemas:

1. Son largos de describir porque involucran diferentes métodos. Esto los hace difíciles de aprender y de evaluar. Los convierte en herramientas incómodas de investigación académica. 
2. Su método es complejo ya que implica una combinación de procesamiento automático y también por juicios del investigador.

3. Los comunicólogos deben de tomar decisiones pragmáticas para limitar el número de análisis realizados y decidir cuál es el mejor.

4. Cualquier método es difícil de evaluar de manera efectiva desde una perspectiva general ya que puede funcionar bien para un tema pero no para otro. Tiene múltiples componentes que deberían de evaluarse por separado y de diferentes formas.

La sociedad actual se desarrolla en un mundo saturado de información. Vivimos rodeados de los medios que nos acompañan desde la mañana hasta la noche. Por lo tanto es importante estudiar el impacto de estos medios en las personas. Pero las herramientas y metodologías de investigación social existentes son muy limitadas para abordar este objetivo de estudio. La mayor parte de estas herramientas son entrevistas y encuestas y son difíciles de manipular para un uso adecuado en experimentos controlados. Es necesario un entorno de investigación actualizado que atraiga a la población. YouTube es una de las páginas web más populares por su enorme difusión. Por lo tanto YouTube puede ser un interesante entorno de investigación. Podemos estudiar cualquier tipo de contenido mediático, desde antisocial, prosocial, comercial, etc... En YouTube podemos encontrar desde imágenes fijas y texto con audio hasta interesantes materiales audiovisuales, series y películas. Una razón que pueden tener los comunicólogos para trabajar con YouTube es la facilidad con la que es posible editar y manipular material para diseños experimentales controlados y su posterior conteo. Se pueden editar y cargar clips audiovisuales y realizar posteriores encuestas sobre ello. Además de un estudio cuantitativo se pueden realizar estudios cualitativos gracias a los comentarios que se pueden incluir en los videos y que pueden ser visibles públicamente. A través de YouTube se pueden 
estudiar los efectos combinados de los medios de comunicación y la retroalimentación de las personas.

YouTube es una herramienta efectiva de investigación. Además es un formato muy accesible para toda la sociedad. Es fácil crear materiales audiovisuales para ser utilizados en experimentos controlados. Gracias a YouTube podemos estudiar las preferencias y respuestas de las personas a un estímulo dado.

Nuestra aportación pretende recopilar las mejores herramientas de análisis de datos de YouTube con las que los comunicólogos y cualquier investigador social puedan trabajar, de forma rápida $\mathrm{y}$ gratuita con miles de datos recopilados de dicha web.

\subsection{Softwares especializados de investigación vinculados a YouTube}

Ciertamente, una de las incertidumbres más comunes al momento de investigar utilizando las redes sociales, se prescribe sobre el uso de motores automatizados capaces de producir datos sobre los criterios seleccionados en el estudio. Dicho de otra forma, hoy en día existen programas gratuitos y webs que facilitan la extracción de datos sin llegar a utilizar códigos de programación, minería de datos ni otras herramientas fuera de las competencias comunes del comunicador.

Si bien la mayoría de estas webs o softwares permiten extraer datos sobre visualizaciones, también existen multiplicidad de métricas como los comentarios emitidos, suscriptores, ingreso estimado, canales similares, posición mundial, frecuencia de publicación de videos, uso de otras redes sociales como Instagram, Facebook o YouTube, entre otros. En efecto, tales datos pudieran servir para determinar la base de objetivos pertenecientes a una hipótesis descriptiva de enfoque cuanticualitativo como también proveer de datos para estudios de marketing.

Dentro de esta amplia serie de opciones, se debe comenzar con las métricas propias generadas por YouTube, en las que se presenta tiempo de visualizaciones, este dato permite conocer el nivel de retención de la audiencia; fuentes de tráfico del canal, esto significa la procedencia de 
las visualizaciones, básicamente la mayoría proviene de las sugerencias realizadas por YouTube en base a previos videos visualizados. Otro dato cosechado por las métricas de la plataforma son los datos demográficos que incluye sexo, área geográfica, edad y dispositivo de visualización (tableta, teléfono inteligente $\mathrm{u}$ ordenador); las ubicaciones de reproducción permiten conocer si se obtiene directamente desde YouTube o si se obtiene desde inserciones en aplicaciones o sitios webs externos. Por último, también provee acceso a YouTube Ads sobre datos de los anuncios e ingresos de publicidad obtenidos de cada video. Aunque esta información es privada para cada canal, diferentes webs han favorecido el establecimiento de criterios y variables conectadas a las analíticas de YouTube como se demuestra en los siguientes casos:

- -Socialblade: Esta web proporciona una gran variedad de datos incorporando información gamificado mediante insignias y rankings (Peñalva, Aguaded \& Torres-Toukoumidis, 2019). Entre los datos iniciales que incluye se encuentran el número de videos cargados, suscriptores, visualizaciones totales, país, tipo de canal y año de creación del canal. Asimismo, se observa el número de visualizaciones de cada video y el rango de ingreso estimado para cada video. Además, tiene una proyección futura del canal donde se muestra la predicción de suscriptores y visualizaciones por cinco años. Es decir, si el análisis se realiza en 2019 se puede observar el rendimiento desde el 2020 hasta el 2024 si mantiene la misma frecuencia de carga de los años anteriores. Contiene una sección detallada de estadísticas organizada mensualmente por dos años y una parte en la que aparecen canales similares. Por último, se presenta el uso de la gamificación con insignias como creación de cuenta hace al menos cinco años, el perfil de Socialblade ha sido visitado frecuentemente, +500 videos cargados en YouTube, +250.000 suscriptores, +75.000.000 visualizaciones, conexión de la cuenta a tres o más redes sociales. El uso de ranking provee 
de un sistema de categorización desde la A++ hasta la D- en comparativa con los canales agregados a esta web donde miden el número de suscriptores, videos publicados e ingresos.

\section{Figura 1. Logo de Socialblade}

\section{.tull SOCIALBLADE}

- Noxinfluencer: Esta web contiene cinco herramientas para YouTube (evaluar los canales, analizar los videos en base a indicadores SEO, comparar canales en base a cinco dimensiones, obtener tendencias en palabras de YouTube y presentar la cantidad de suscriptores del canal en tiempo real). El primero de ellos, evaluar canales de YouTube, se basa en una calculadora que permite conocer la ganancia diaria, mensual y anual estimada por el canal a través del coste por cada mil impresiones que deben pagar los anunciantes. En cuanto al análisis de los indicadores SEO para cada video se obtiene datos complementarios a las otras plataformas como el porcentaje entre "me gusta" y "no me gusta”, etiquetas eíndice de interacción (proporción de la sumatoria de "me gusta" y comentarios). La plataforma permite comparar hasta tres canales en base a cinco dimensiones reflejados con un diagrama de red, estas cinco dimensiones son: crecimiento de suscriptores, abundancia de las redes sociales, promedio de rendimiento de los videos, tasa de interacción y secuencia de actualización de video. Respecto a herramientas de palabras clave, se parece a la información de Google Analytics pero para YouTube. Es decir, el motor de coincidencia busca ciertas palabras clave según las tendencias en YouTube. Desafortunadamente, este servicio solo está presente para Estados Unidos, Canadá, Corea del Sur, Arabia Saudí, India, Taiwán, Reino Unido, Indonesia, España, Portugal 
y Japón. Para culminar, y quizás la siendo la herramienta menos útil para la investigación, se trata del conteo de suscriptores en vivo y en tiempo real que se actualiza cada cuatro segundos.

- Exportcomments: La versión gratuita de la web permite extraer hasta 100 comentarios, incluyendo comentarios anidados que se realizan en cualquier canal de YouTube. Esta herramienta es sumamente útil ya que posterior a la extracción se puede formalizar análisis del discurso jerarquizándolos según un compendio de criterios y restricciones. Esto implica que YouTube no solo es solo el video, todos los elementos que circundan la interacción también pueden servir de objeto de estudio.

- Socialtracker: Plataforma web que elabora un reporte automatizado similar a Socialblade pero menos detallado. El valor agregado que contiene es el indicador de metas en el que aparece la meta de suscriptores y de visualizaciones para el año siguiente. Esta web simplifica la información de la anterior. En otras palabras, si los indicadores determinados para la investigación de canales de YouTube no conllevan profundidad, entonces esta herramienta es ideal para el análisis.

- Statsheep: Página web esencial para realizar comparativas entre dos canales, permitiendo vislumbrar las diferencias que existen respecto al número de suscriptores, visualizaciones y videos cargados. También contienen su propio ranking obtenido en base a características como el crecimiento, viralización y visualizaciones.

- Watching.today: Web utilizada para estudios genéricos sobre los videos que se encuentran en tendencia en diferentes temáticas de algunos países. Si bien Ecuador no está dentro de los países latinoamericanos evaluados en esta herramienta, se ubica Argentina, Brasil, Chile, Colombia, México y Perú. Respecto a las temáticas se localizan autos y vehículos; comedia; educción; entretenimiento; películas y animación; juegos; estilo; música; noticias y políticas; personas y blogs; animales y mascotas; 
ciencias y tecnología; deportes y viajes. Esta web puede servir para estudio a nivel macro sobre comparativas entre países respecto al contenido y su posible remarcación de las preferencias de la audiencia.

- Ytcount: Similar a Watching.today. Sin embargo, en este caso aparece Ecuador y otorga métricas para cada canal. Las métricas incorporadas son crecimiento diario de visualizaciones $\mathrm{y}$ suscriptores, número de videos cargados y estimación de ganancia por publicidad. Además, permite comparar el número de seguidores entre dos canales.

\section{Figura 2. Logo de Ytcount}

\section{or YT COUNT}

- InspectSocial: La web provee de información gratuita sobre estadísticas y proyecciones. A diferencia de las otras, suministra la diferencia proporcional diaria de suscriptores, visualizaciones y videos. Esto implica que se puede verificar con mayor claridad, los cambios, siendo progresos o retrocesos en cada cuenta de YouTube.

- Screamingfrog:Único software de descarga dentro de estelistado. En la coyuntura de estar realizando un trabajo de investigación multidisciplinario con un equipo informático, este software genera una serie de indicadores importantes para evaluar los diferentes enlaces, incluso los de YouTube, especialmente en la sección que se halla bajo el video. Los indicadores frecuentes utilizados son enlaces rotos, metadatos, urls bloqueadas, rastreo sobre contenido duplicado, etc. 


\section{Conclusiones}

Nuestro capítulo de libro se centra en cómo YouTube se puede convertir en una herramienta de investigación para investigadores en comunicación. Esta investigación puede ser de corte cuantitativo y cualitativo. YouTube es el formato más apropiado para trabajar con la población en diseños experimentales y transversales.

Hemos presentado varios estudios en donde YouTube sirvió como escenario de investigaciones de todo tipo, unos de tipo cuantitativo, de análisis de contenido de los comentarios y otros de diseños experimentales. Cada investigación mostrada trabaja con dispositivos de medición basados en YouTube. Así la configuración de YouTube permite investigar sistemáticamente diversos aspectos a analizar (las visualizacionesy los comentarios principalmente). La naturalezagráfica y textual de YouTube así como su plasticidad nos permiten ejercer un amplio control sobre las imágenes, videos y comentarios textuales. Las futuras investigaciones en YouTube son muy prometedoras y abren nuevos horizontes para la investigación de todo tipo (cuantitativa, cualitativa o experimental) en comunicación.

La configuración de YouTube proporciona un entorno de investigación ecológicamente válido, ya que es un canal de medios muy accesible a toda la población y a través del cual las personas pueden visualizar y enviar comentarios sobre el contenido que han visto y que es visible para otras personas. YouTube también favorece el anonimato y por ello las personas se expresan sin miedo ni ataduras. El uso de los alias y de diversas jergas que no se ajustan a un vocabulario fortalecen esta idea. Las características de YouTube brindan muchas oportunidades para realizar muchas más investigaciones en el ámbito de la comunicación por parte de empresas, instituciones o universidades. Si conociéramos el perfil sociodemográfico, su perfil de actividad o el dispositivo desde donde se conecta de los usuarios de YouTube en investigaciones futuras se podrían analizar datos desglosados y estudiar los efectos diferenciales en los resultados. 
Las distintas opciones de ajusten en el contenido, el formato y la información de los usuarios hacen que YouTube sea muy útil para una amplia gama de temas de investigación en comunicación (por ejemplo, el estudio de la publicidad, el ciberacoso, los movimientos sociales, temas políticos, etc...).

La mayoría de las investigaciones en comunicación estaban basadas en la exposición a los medios en encuestas sobre cuántas horas dedicaban a ver la televisión, ver películas, jugar a videojuegos o horas conectado a Internet. Había otras investigaciones más específicas sobre encuestas sobre exposición a contenidos violentos en películas, series o videojuegos. Tales investigaciones han sido útiles en investigaciones pasadas, pero se quedan cortas en el panorama mediático actual.

Los contenidos audiovisuales están disponibles en diversos formatos (por ejemplo, una película la podemos ver en la televisión, en YouTube desde nuestro ordenador de mesa, en un portátil, en la Tablet o en el Smartphone). Por lo tanto, es necesario herramientas para evaluar su exposición y las preferencias para los contenidos específicos. Nuestro capítulo de libro puede servir a ver más allá del bosque y ofrecemos herramientas para el análisis desde YouTube. Concluimos que las herramientas que ofrecemos son idóneas para realizar investigaciones en el campo de la comunicación desde el ámbito cuantitativo, cualitativo o experimental.

\section{Referencias}

Baron, S., Conway, T., \& Warnaby, G. (2010). Relationship marketing: A consumer experience approach. EEUU: Sage Publications.

Bowyer, B. T., Kahne, J. E., \& Middaugh, E. (2017). Youth comprehension of political messages in YouTube videos. New Media \& Society, 19(4), 522-541. https://doi.org/10.1177/1461444815611593

Burgess, J. E. y Green, J.B. (2009). YouTube: online video and participatory culture. Cambridge: Polity Press. 
Burke, S. C., \& Snyder, S. L. (2008). YouTube: An Innovative Learning Resource for College Health Education Courses. International Electronic Journal of Health Education, 11, 39-46. https://bit.ly/2sZbpZx

Chen, C. P. (2013). Exploring personal branding on YouTube. Journal of Internet Commerce, 12(4), 332-347. https://doi.org/10.1080/15332861.2013.859041

Chung, J. E. (2015). Antismoking campaign videos on YouTube and audience response: Application of social media assessment metrics. Computers in Human Behavior, 51, 114-121. https://doi.org/10.1016/j.chb.2015.04.061

Costa-Sánchez, C., \& Túñez-López, M. (2019). Contenidos audiovisuales en social media. Análisis comparativo de Facebook y YouTube. Fonseca, Journal of Communication, 19, 223-236. https://bit.ly/36ArVh5

Desai, T., Shariff, A., Dhingra, V., Minhas, D., Eure, M., \& Kats, M. (2013). Is content really king? An objective analysis of the public's response to medical videos on YouTube. PLoS One, 8(12), e82469. https://doi.org/10.1371/journal. pone.0082469

Díaz Arias, R. (2009). El vídeo en el ciberespacio: usos y lenguaje. Comunicar: Revista científica iberoamericana de comunicación y educación, (33), 63-71. https://doi.org/10.3916/c33-2009-02-006

Fan, W., \& Gordon, M. D. (2014). The power of social media analytics. Communications of the ACM, 57, 74-81. https://bit.ly/35CH9ki

García-Ruiz, R., Pérez-Rodríguez, A., \& Torres-Toukoumidis, A. (2018). Educar para los nuevos medios. Claves para el desarrollo de la competencia mediática en el entorno digital. Abya-Yala: Ecuador.

Jaffar, A. A. (2012). YouTube: An emerging tool in anatomy education. Anatomical sciences education, 5(3), 158-164. https://doi.org/10.1002/ase.1268

Jansson, A., \& Lindell, J. (2018). Media studies for a mediatized world: rethinking media and social space. Media and Communication, 6(2), 1-4. https://doi. org/10.17645/mac.v6i2.1495

Khosravinik, M., \& Unger, J. W. (2016). Critical discourse studies and social media: Power, resistance and critique in changing media ecologies. En Ruth Wodak y Michael Meyer (Ed.) Methods of critical discourse studies, Sage, London, pp. 205-233. 
Lewis, S. P., Heath, N. L., Sornberger, M. J., \& Arbuthnott, A. E. (2012). Helpful or harmful? An examination of viewers' responses to nonsuicidal self-injury videos on YouTube. Journal of Adolescent Health, 51, 380-385. https://doi. org/10.1016/j.jadohealth.2012.01.013

Loader, B. D., \& Mercea, D. (2011). Networking democracy? Social media innovations and participatory politics. Information, Communication $\&$ Society, 14.6), 757-769. https://doi.org/10.1080/1369118X.2011.592648

Lopes, I., Guarda, T., Victor, J. A., \& Vázquez, E. G. (2020). The Influence of YouTubers in Consumer Behavior. En Marketing and Smart Technologies (pp. 49-58). Springer, Singapore.

Madathil, K. C., Rivera-Rodriguez, A. J., Greenstein, J. S., \& Gramopadhye, A. K. (2015). Healthcare information on YouTube: a systematic review. Health informatics journal, 21(3), 173-194. https://doi.org/10.1177/1460458213512220

Moreira Aguirre, D. G., Pérez Samaniego, S. J., Altamirano Benítez, V. P., \& Marín-Gutiérrez, I. (2019). Citizen Influence in Social Media for Democratization of Communication. En Communication: Innovation \& Quality (pp. 207-228). Springer, Cham.

O’Reilly, T. (2006). Qué es Web 2.o. Patrones del diseño y modelos del negocio para la siguiente generación del software. Boletín de la Sociedad de la Información: Tecnología e Innovación, (3), 177-201. https://bit.ly/2RWrgQQ

Owen, B. M. (2000). The Internet challenge to television. Massachusetts: Harvard. Parratt Fernández, S. (2002). La glocalización de la comunicación. Ámbitos, (7-8), 151-163. https://bit.ly/2RFQfZN

Peñalva, S., Aguaded, I., \& Torres-Toukoumidis, Á. (2019). La gamificación en la universidad española. Una perspectiva educomunicativa. Revista Mediterránea de Comunicación/Mediterranean Journal of Communication, 10(1), 245-256. https://bit.ly/36yX44C

Pérez Dasilva, J. A. y Santos Díez, T. (2009). Las televisiones locales del País Vasco en Internet. Revista Latina de Comunicación Social, (64), 192-202. https:// bit.ly/2vBU7mb

Ritzer, G. (2001). Teoría sociológica clásica. Madrid: McGraw-Hill. https:// go.aws/2OgcJyy 
Robinson, J. P. y Geoffrey, G. (1997). Time for life: The surprising ways Americans use their time. Pennsylvania: Pennsylvania State Press.

Rovira-Collado, J. (2017). Booktrailer y Booktuber como herramientas LIJ 2.0 para el desarrollo del hábito lector. Investigaciones sobre Lectura, (7), 55-72. https://bit.ly/35vOKkH

Ruiz-San-Miguel, F.J.; Hinojosa-Becerra, M. y Marín-Gutiérrez, I. (2016). Teaching with videos on YouTube: Case study of the subject technical and editing still image. 11th Iberian Conference on Information Systems and Technologies (CISTI). DOI: 10.1109/CISTI.2016.7521384.

Sarker, A., O’Connor, K., Ginn, R., Scotch, M., Smith, K., Malone, D., \& Gonzalez, G. (2016). Social media mining for toxicovigilance: automatic monitoring of prescription medication abuse from Twitter. Drug safety, 39(3), 231-240. http://dx.doi.org/10.1007/s40264-016-0497-7.

Schneider, C. J. (2016). Music videos on YouTube: Exploring participatory culture on social media. En Symbolic Interactionist Takes on Music (pp. 97-117). Emerald Group Publishing Limited.

Sharma, M., Yadav, K., Yadav, N., \& Ferdinand, K. C. (2017). Zika virus pandemicanalysis of Facebook as a social media health information platform. American journal of infection control, 45(3), 301-302. https://doi.org/10.1016/j. ajic.2016.08.022

Tan, E. (2013). Informal learning on YouTube: Exploring digital literacy in independent online learning. Learning, Media and Technology, 38(4), 463-477. https://doi.org/10.108o/17439884.2013.783594.

Thelwall, M., Buckley, K., \& Paltoglou, G. (2011). Sentiment in Twitter events. Journal of the American Society for Information Science and Technology, 62, 406-418. https://doi.org/10.1002/asi.21462

Tijdens, K., \& Steinmetz, S. (2016). Is the web a promising tool for data collection in developing countries? An analysis of the sample bias of 10 web and face-to-face surveys from Africa, Asia, and South America. International Journal of Social Research Methodology, 19, 461-479. https://doi.org/10.10 80/13645579.2015.1035875 
Torres-Toukoumidis, Á., Ramírez-Montoya, M. S., \& Romero-Rodríguez, L. M. (2018). Valoración y evaluación de los Aprendizajes Basados en Juegos (GBL) en contextos e-learning. Education in the Knowledge Society, 19(4), 109-128.

Way, L. C. (2015). YouTube as a site of debate through populist politics: the case of a Turkish protest pop video. Journal of Multicultural Discourses, 10(2), 180-196. https://doi.org/10.1080/17447143.2015.1041965

Wolton, D. (2000). Internet, ¿̇y después? Barcelona: Gedisa.

$\mathrm{Wu}$, K. (2016). YouTube marketing: Legality of sponsorship and endorsements in advertising. JL Bus. \& Ethics, (22), 59-91. https://bit.ly/39MGuQx

YouTube (2012). Sala de prensa. Estadísticas 2020. http://www.YouTube.com/t/ press_statistics. 
